\title{
Global connectivity and expansion: long cycles and factors in $f$-connected graphs
}

(Extended version)

\author{
Stephan Brandt, Technische Universität Ilmenau \\ Hajo Broersma, Universiteit Twente \\ Reinhard Diestel, Universität Hamburg \\ Matthias Kriesell, Universität Hannover
}

2 July 2002

\begin{abstract}
Given a function $f: \mathbb{N} \rightarrow \mathbb{R}$, call an $n$-vertex graph $f$-connected if separating off $k$ vertices requires the deletion of at least $f(k)$ vertices whenever $k \leq(n-f(k)) / 2$. This is a common generalization of vertex connectivity (when $f$ is constant) and expansion (when $f$ is linear). We show that an $f$-connected graph contains a cycle of length linear in $n$ if $f$ is any linear function, contains a 1-factor and a 2-factor if $f(k) \geq 2 k+1$, and contains a Hamilton cycle if $f(k) \geq 2(k+1)^{2}$. We conjecture that linear growth of $f$ suffices to imply hamiltonicity.
\end{abstract}

\section{Introduction: the notion of $f$-connectedness}

In his survey on tree-width, tangles and graph minors, Reed [21] starts out by considering the global connectivity properties of a hexagonal grid: he observes that, although any such grid is barely 2-connected, the number of vertices needed to disconnect a large part from the rest is large: it grows at least as $\sqrt{k}$ with the size $k$ of that part. Our aim in this paper is to define this notion of global connectivity more formally, to see how it relates to other graph invariants, and in particular to ask what 'richness' properties (such as hamiltonicity, or the existence of other global substructures) it can imply.

Given a graph $G$, let us call a pair $(A, B)$ of proper subsets of $V(G)$ a separation of $G$ if $A \cup B=V(G)$ and $G$ has no edge between $A \backslash B$ and $B \backslash A$. Let $f: \mathbb{N} \backslash\{0\} \rightarrow \mathbb{R}$ be a function (usually non-decreasing). 
Definition 1.1 $G$ is called $f$-connected if every separation $(A, B)$ of $G$ with $|A \backslash B| \leq|B \backslash A|$ satisfies $|A \cap B| \geq f(|A \backslash B|)$.

Thus, for small $k$, splitting off $k$ vertices requires a separator of size at least $f(k)$.

For constant functions $f: k \mapsto c \in \mathbb{N}, f$-connectedness reduces to the traditional notion of $c$-connectedness. Complete graphs are $f$-connected for every $f$ (because they have no separations), and grids are $f$-connected for $f: k \mapsto \sqrt{2 k}$ (see $[6,22])$.

An obvious equivalent way to view $f$-connectedness is as an expansion property, ie. in terms of an isoperimetric inequality (such as (1) below). Isoperimetric inequalities have been investigated mainly for specific classes of graphs (such as higher-dimensional grids and cubes; see e.g. [6], and [9] for references), while the focus in the study of expanders has largely been to construct (sparse) expanders with certain desired properties [9, 15].

Our aim here differs from both these: rather than trying to determine for which $f$ some specific graphs are $f$-connected, or to construct $f$-connected graphs for certain specific $f$, we shall think of $f$ as given but arbitrary, and then try to relate the corresponding property of $f$-connectedness to other graph invariants. This has two aspects:

- What properties or substructures of a graph $G$ can we force by assuming that $G$ is $f$-connected?

- What assumptions will force a graph $G$ to be $f$-connected or to have an $f$-connected subgraph (or minor etc)?

We will mainly be concerned here with the first of these aspects and only touch briefly on the second. The second aspect has been studied in more depth by Rempel [22], who investigated for which functions $f$ the assumption of high average degree or chromatic number can force $G$ to have large $f$ connected subgraphs.

Our graph-theoretical notation is that of [12]. In particular, if $S$ is a set of vertices in a graph $G=(V, E)$, we denote by $N_{G}(S)=N(S)$ the set of neighbours in $G-S$ of vertices in $S$. Initial, middle and final segments of a path $P$ are denoted as $P y, x P y$ and $x P$, respectively, if they start at $x$ and/or end at $y$. Unless otherwise stated, $f$ will always denote an arbitrary non-decreasing function $\mathbb{N} \backslash\{0\} \rightarrow \mathbb{R}$.

Throughout the paper, we shall use freely the following two reformulations of $f$-connectedness as an expansion property. For

$$
\bar{S}:=V \backslash(S \cup N(S)),
$$


clearly $G$ is $f$-connected if and only if

$$
|N(S)| \geq f(|S|) \text { for every } S \subseteq V \text { with } 0<|S| \leq|\bar{S}|
$$

(Rephrased like this, $f$-edge-connectedness could be defined correspondingly in terms of edge boundaries.)

Put another way, if $|G|=: n$ and $G$ is $f$-connected, then a non-empty set $S \subseteq V$ can satisfy $|N(S)|<f(|S|)$ only if $|S|>|\bar{S}|=n-|N(S)|-|S|$, or equivalently if $|N(S)|>n-2|S|$. Thus, $G$ is $f$-connected if and only if every $S \subseteq V$ satisfies

$$
|N(S)| \geq \min \{f(|S|), n-2|S|+1\} .
$$

Our paper is organized as follows. We start out in Section 2 with our main results, establishing upper bounds on the growth rate of $f$ needed to force a Hamilton cycle or other long cycle in a given graph. In Section 3 we do the same for 1 -factors and 2-factors. Section 4 gives some brief indications of the kind of growth rate of $f$ that can be expected to force standard substructures (such as given subgraphs or minors), and suggests some open problems. In Section 5 we relate $f$-connectedness for regular graphs and linear $f$ to the traditional concept of an $(n, d, c)$-expander, and note a lower bound in terms of eigenvalues. As an application of our main results we deduce the existence of long cycles and factors in Ramanujan graphs.

\section{$2 \quad$ Forcing long cycles}

It is well known that random graphs contain long cycles, indeed Hamilton cycles, even when they are relatively sparse. On the other hand, they are well-expanding. Our aim in this section is to relate these two properties directly: we shall prove that linear $f$-connectedness always forces a cycle of length linear in the order of the graph, and that quadratic $f$-connectedness can force it to be hamiltonian.

Theorem 2.1 If $f(k) \geq 2(k+1)^{2}$ for all $k \in \mathbb{N}$, then every $f$-connected graph of order $n \geq 3$ is hamiltonian.

We do not believe that quadratic growth of $f$ is needed in Theorem 2.1, but expect that some linear growth will do:

Conjecture 2.2 There exists a function $f(k)=O(k)$ such that every $f$ connected graph of order $n \geq 3$ is hamiltonian. 
We shall see later (Section 4) that Conjecture 2.2 follows from Chvátal's toughness conjecture [10].

Since bipartite graphs $K_{n, n-1}$ are not hamiltonian but $f$-connected for $f: k \mapsto 2 k$ when $n$ is odd, the strongest possible version of Conjecture 2.2 would be the following:

Conjecture 2.3 If $f(k) \geq 2 k+1$ for all $k \in \mathbb{N}$, then every $f$-connected graph of order $n \geq 3$ is hamiltonian.

Although we cannot prove either of these conjectures, we can show that an $f$-connected graph with $f(k) \geq 2 k$ contains a cycle of length at least $3 n / 4$. This is the case $c=2$ of the following more general result:

Theorem 2.4 Let $f(k) \geq$ ck for some real number $c \geq 2$ and all $k \in \mathbb{N}$, and let $G$ be an $f$-connected graph of order $n \geq 3$. Then $G$ has a cycle of length at least $\frac{c+1}{c+2} n$.

For $c=2$, this improves the lower bound of $5 n / 9$ obtained by Rempel [22].

We shall prove Theorems 2.1 and 2.4 by the same method. This method does not allow an extension of Theorem 2.4 to $c<2$. For $c<2$ we can still show that $f$-connected graphs have cycles of linear length, but only with a smaller multiplicative constant:

Theorem 2.5 Let $f(k) \geq$ ck for some real number $c \in(0,2)$ and all $k \in \mathbb{N}$, and let $G$ be an $f$-connected graph of order $n>1+\frac{3}{c}$. Then $G$ has a cycle of length at least $\left(\frac{c}{c+2}\right)^{2} n$.

We remark that the lower bound on $n$ in Theorem 2.5 is necessary: there can be trees of order up to $1+\frac{3}{c}$ that are $f$-connected with $f(k)=c k$. See the remark following Lemma 2.10.

In our proofs we shall use the following tool due to Pósa [20]. Let $P$ be a path in a graph $G$, say from $u$ and $v$. Given a vertex $x \in P$, we write $x^{-}$for the vertex preceding $x$ on $P$, and $x^{+}$for the vertex following $x$ on $P$ (whenever these exist). Similarly for $X \subseteq V(P)$ we put

$$
X^{-}:=\left\{x^{-} \mid x \in X\right\} \quad \text { and } \quad X^{+}:=\left\{x^{+} \mid x \in X\right\} .
$$

If $x \in P-u$ is a neighbour of $u$ in $G$, then $P-x x^{-}+u x$ (which is a path in $G$ with vertex set $V(P)$ ) is said to have been obtained from $P$ by an elementary exchange fixing $v$ (Fig. 1). A path obtained from $P$ by a (possibly empty) 
sequence of elementary exchanges fixing $v$ is a path derived from $P$. The set of starting vertices of paths derived from $P$, including $u$, will be denoted by $S(P)$. As all paths derived from $P$ have the same vertex set as $P$, we have $S(P) \subseteq V(P)$.

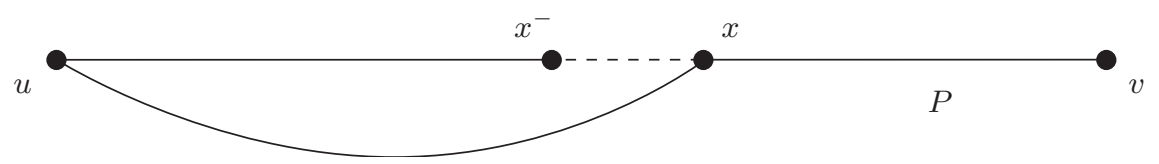

Figure 1: An elementary exchange applied to the path $P$

The following lemma is a one-sided variant of Pósa's Lemma [20]; see also [7, Lemma 6.3].

Lemma 2.6 Let $G$ be a graph, let $P=u \ldots v$ be a longest path in $G$, and put $S:=S(P)$. Then $N_{G}(S) \subseteq S^{-} \cup S^{+}$.

Proof. Let $x \in S$ and $y \in N(x)$ be given; we show that $y \in S^{-} \cup S \cup S^{+}$. As $x \in S$ there is a path $Q=x \ldots v$ derived from $P$. Then $y \in V(Q)=V(P)$, because $Q$ (like $P$ ) is a longest path; let $z$ denote the predecessor of $y$ on $Q$.

Suppose that $y \notin S^{-} \cup S \cup S^{+}$. Then each of the (one or two) edges $e \in P$ at $y$ lies on every path derived from $P$ (and in particular on $Q$ ), because in any elementary exchange in which $e$ is first deleted, its two ends (including $y$ ) would have become members of $S$ and of $S^{-} \cup S^{+}$, respectively. Hence $z \in\left\{y^{-}, y^{+}\right\}$. But $Q+x y-y z$ is obtained from $Q$ by an elementary exchange, which puts $z$ in $S$ and $y$ in $S^{-} \cup S^{+}$.

Lemma 2.7 Let $G$ be a graph, let $P=u \ldots v$ be a longest path in $G$, and put $S:=S(P)$. Then $G$ has a cycle containing $S \cup N(S)$.

Proof. Let $y$ be the last vertex of $P$ in $N(S)$. Then all the vertices from $S \cup N(S)$ lie on $P y$, because any vertex of $y P$ in $S$ would differ from $v$ and hence have its successor on $P$ in $N(S)$. Let $x \in S$ be a neighbour of $y$ in $G$, and let $Q=x \ldots v$ be derived from $P$. As in the proof of Lemma 2.6, all the edges of $y P$ are still edges of $Q$, so $y Q=y P$. Thus $S \cup N(S) \subseteq V(P y)=V(Q y)$, and $Q y x$ is a cycle in $G$. 


\section{Proof of Theorem 2.4.}

Let $P=u \ldots v$ be a longest path in $G$, and put $S:=S(P)$.

Suppose first that $|S| \leq n /(c+2)$. Then

$$
|N(S)| \underset{(1)}{\geq} \min \{c|S|, n-2|S|+1\} \geq c|S|,
$$

which by Lemma 2.6 implies the contradiction of

$$
3|S|-1 \geq\left|S^{+} \cup S \cup S^{-}\right| \geq|S \cup N(S)| \geq|S|+c|S| \geq 3|S| .
$$

(The -1 comes from the fact that $u \in S$ while $u^{-}$does not exist.)

We may therefore assume that $|S|>n /(c+2)$. Choose a subset $S^{\prime} \subseteq S$ with

$$
\left|S^{\prime}\right|=\left\lceil\frac{n}{c+2}\right\rceil \text {. }
$$

Then

$$
\left|S^{\prime} \cup N\left(S^{\prime}\right)\right| \underset{(1)}{\geq} \min \left\{(c+1)\left|S^{\prime}\right|, n-\left|S^{\prime}\right|+1\right\} \geq \frac{c+1}{c+2} n .
$$

Now the cycle from Lemma 2.7, which contains $S \cup N(S) \supseteq S^{\prime} \cup N\left(S^{\prime}\right)$, has the desired length.

\section{Proof of Theorem 2.1.}

Let $G$ be an $f$-connected graph with $|G|=: n \geq 3$ and $f(k) \geq 2(k+1)^{2}$ for all $k \in \mathbb{N}$. We first show that $G$ contains a longest path $P=u \ldots v$ with

$$
\sqrt{2 n}-2<d(u) \leq d(v) .
$$

Choose $P=u \ldots v$ among all the longest paths in $G$ with $d(u) \leq d(v)$ so that $d(u)$ is maximum, and subject to this with $d(v)$ maximum. Put $S:=S(P)$. By Lemma 2.6 and (1), we have

$$
2|S| \geq\left|S^{-} \cup S^{+}\right| \geq|N(S)| \geq \min \left\{2(|S|+1)^{2}, n-2|S|+1\right\}
$$

and hence $2|S| \geq n-2|S|+1$, giving

$$
|S| \geq\lceil(n+1) / 4\rceil \geq\lfloor\sqrt{n / 2}\rfloor .
$$

Consider a subset $S^{\prime} \subseteq S$ with $\left|S^{\prime}\right|=\lfloor\sqrt{n / 2}\rfloor$. By (1), we have

$$
\left|N\left(S^{\prime}\right)\right| \geq \min \left\{2\left(\left|S^{\prime}\right|+1\right)^{2}, n-2\left|S^{\prime}\right|+1\right\} .
$$


As $2\left(\left|S^{\prime}\right|+1\right)^{2}>n$ by the choice of $S^{\prime}$, this yields

$$
\left|N\left(S^{\prime}\right)\right| \geq n-2\left|S^{\prime}\right|+1>n-\sqrt{2 n},
$$

so some vertex $s \in S^{\prime}$ has degree at least

$$
\frac{\left|N\left(S^{\prime}\right)\right|}{\left|S^{\prime}\right|}>\frac{n-\sqrt{2 n}}{\sqrt{n / 2}}=\sqrt{2 n}-2 .
$$

As $s \in S$, there is a longest path $Q=s \ldots v$ in $G$. Thus if (2) fails and hence $d(u)<d(s)$, then the existence of $Q$ contradicts the choice of $P$. This completes the proof that $P$ satisfies (2).

We next show that $G$ contains a cycle $C$ with $V(C)=V(P)$. Since $G$ is connected and $P$ is a longest path, this cycle must then be a Hamilton cycle. If $u v \in E(G)$ then $C:=P v u$ will do, so let us assume that $u v \notin E(G)$.

Let $w$ be the first vertex on $P$ such that some $x \in\{u, v\}$ has at least half its neighbours on $P w$. Put $P_{1}:=P w$ and $N_{1}:=N(x) \cap V\left(P_{1}\right)$, and let $S_{1}:=N_{1}^{-}$. Note that $u \notin N_{1}$ since $u v \notin E(G)$, so

$$
\left|S_{1}\right|=\left|N_{1}\right| \geq d(x) / 2>\sqrt{n / 2}-1
$$

by (2). Now let $y$ be such that $\{x, y\}=\{u, v\}$, put $P_{2}:=w P$ and $N_{2}:=$ $N(y) \cap V\left(P_{2}\right)$, and let $S_{2}:=N_{2}^{+}$. Note that, by the choice of $w$, at least half the neighbours of $y$ lie in $N_{2}$. Thus as before, $u v \notin E(G)$ and (2) imply that

$$
\left|S_{2}\right|=\left|N_{2}\right| \geq d(y) / 2>\sqrt{n / 2}-1 .
$$

For $i=1,2$ we have

$$
\left|N\left(S_{i}\right)\right| \geq \min \left\{2\left(\left|S_{i}\right|+1\right)^{2}, n-2\left|S_{i}\right|+1\right\}
$$

by (1). Since $2\left(\left|S_{i}\right|+1\right)^{2}>n$, this means that $\left|N\left(S_{i}\right)\right|>n-2\left|S_{i}\right|$. Applying this with $S_{i}$ denoting the smaller of the sets $S_{1}$ and $S_{2}$, we obtain

$$
\left|N\left(S_{i}\right)\right|>n-2\left|S_{i}\right| \geq n-\left(\left|S_{1}\right|+\left|S_{2}\right|\right) .
$$

So this $S_{i}$ must have a neighbour in the other $S_{j}$, ie. $G$ contains an edge $e$ between $S_{1}$ and $S_{2}$. Figure 2 shows how this edge can be used to obtain the desired cycle $C$ in each of the two cases of either $x=u$ and $y=v$ or $x=v$ and $y=u$. 


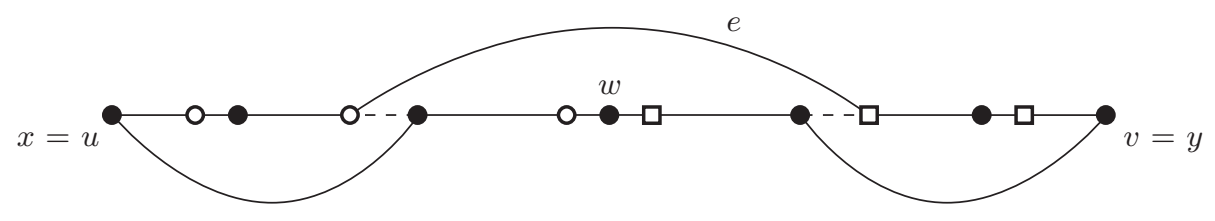

$$
\text { ○ }=S_{1} \quad \square=S_{2}
$$

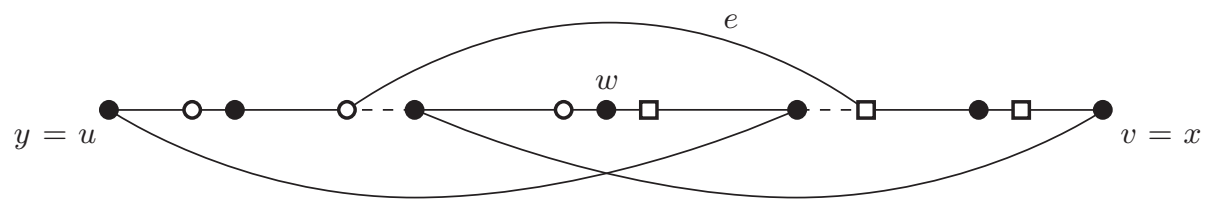

Figure 2: Finding the cycle $C$ in the proof of Theorem 2.1

We finally turn to the proof of Theorem 2.5. We start with another Lemma.

Lemma 2.8 Let $T$ be a tree of order at least 3 , and $r \in T$ a vertex. Then $T$ contains a path $P=r \ldots s$ where $s$ is not a leaf, such that $V(T-P)$ is a union of two disjoint sets $A$ and $B$, possibly empty, whose sizes differ by at most 1 and which are not joined by an edge of $T$.

Proof. Let $P=r \ldots s$ and $A, B$ be as above ignoring the size condition, but with $|A|$ and $|B|$ as equal as possible. Suppose that $|A| \leq|B|-2$. If $s$ is adjacent to a leaf in $B$, then moving this leaf to $A$ decreases the difference between $|A|$ and $|B|$, a contradiction. If $s$ has a neighbour $t \in B$ that is not a leaf, the extended path $P$ st with $V(T-P s t)$ split into $A$ and $B \backslash\{t\}$ yields a contradiction. So $s$ has no neighbour in $B$. Then $s \neq r$, and if $P=r s$ then $r$ is not a leaf. So $P^{\prime}:=P-s$ is a path not ending in a leaf, and $V\left(T-P^{\prime}\right)$ splits into the sets $A \cup\{s\}$ and $B$ (a contradiction).

Lemma 2.9 Suppose that $f(k) \geq$ ck for some real number $c>0$ and all $k \in \mathbb{N}$, and let $G$ be an $f$-connected graph of order $n \geq 3$. Then $G$ contains a path of order at least $\frac{c}{c+2} n$.

Proof. As $f(k)>0$ for all $k>0$, we know that $G$ is connected. Let $T \subseteq G$ be a DFS-tree (a normal spanning tree in [12]), with root $r$ say. By Lemma 2.8 there is a path $P=r \ldots s$ in $T$ such that $s$ is not a leaf and 
$V(T-P)$ is a union of disjoint sets $A, B$ that are not joined by an edge of $T$ and whose sizes $|A| \leq|B|$ differ by at most 1 . Since $T$ is normal in $G$, we have $N_{G}(A) \subseteq V(P)$ : as $P$ is down-closed in the tree-order of $T$ associated with $r$, every component of $T-P$ and hence $A$ is up-closed, so any neighbour outside $A$ of a vertex $a \in A$ lies below $a$ and therefore not in $B$ (since $B$ too is up-closed).

As $s$ is not a leaf, $P$ is not a longest path in $G$. Thus if $p$ denotes the order of a longest path in $G$, then

$$
|A| \geq \frac{1}{2}(n-|P|-1) \geq \frac{1}{2}(n-(p-1)-1)=\frac{1}{2}(n-p) .
$$

Since $N(A) \subseteq V(P)$, this implies

$$
p>|P| \geq|N(A)| \geq c|A| \geq \frac{1}{2} c(n-p),
$$

giving $p>\frac{c}{c+2} n$ as desired.

Lemma 2.10 Suppose that $f(k) \geq$ ck for some real number $c>0$ and all $k \in \mathbb{N}$, and let $G$ be an $f$-connected graph of order $n>1+\frac{3}{c}$. If $n \geq 3$, then $G$ contains a cycle.

Proof. As $f(k)>0$ for all $k>0$, we know that $G$ is connected. Suppose the lemma fails, ie. that $G$ is a tree. Orient every edge of $G$ towards where more vertices of $G$ lie, breaking ties arbitrarily, and let $v$ be a sink. We claim that $V(G-v)$ is a union of disjoint sets $A$ and $B$ such that $\frac{1}{3}(n-1) \leq|A| \leq|B|$.

To show this, let us collect components of $G-v$ greedily (large sizes first) until we have collected at least $\frac{1}{3}(n-1)$ vertices, and call this set of vertices $C$. Then $\frac{1}{3}(n-1) \leq|C|<\frac{2}{3}(n-1)$, and we let $A$ be the smaller of the sets $C$ and $V(G-v) \backslash C$, and $B$ the larger.

Now $|N(A)|=|\{v\}|=1$, so for $k:=|A|$ we have $c k \leq f(k) \leq 1$ and hence $\frac{1}{3}(n-1) \leq k \leq 1 / c$. Thus $n \leq 1+\frac{3}{c}$, contrary to our assumption.

We remark that the requirement of $n>1+\frac{3}{c}$ in Lemma 2.10 is indeed necessary if we want linear $f$-connectedness to force the existence of a cycle. Indeed, for every $c>0$ with $1 / c$ integral there is a tree of order $n=1+\frac{3}{c}$ that is $f$-connected with $f(k)=c k$ : take any tree with a vertex of degree 3 whose deletion yields three subtrees of order $1 / c$.

Lemma 2.11 Suppose that $f(k) \geq$ ck for some real number $c>0$ and all $k \in \mathbb{N}$, and let $G$ be an $f$-connected graph of order $n>1+\frac{3}{c}$. Let $p$ denote the order of a longest path in $G$. If $n \geq 3$, then $G$ contains a cycle of length at least $\frac{c}{c+2} p$. 
Proof. Let $P$ be a longest path in $G$. Let $S_{1}$ denote the set of the first $\left\lfloor\frac{p}{c+2}+1\right\rfloor$ vertices of $P$, let $S_{2}$ denote the set of its last $\left\lfloor\frac{p}{c+2}+1\right\rfloor$ vertices, and put $P^{\prime}:=P-S_{1}-S_{2}$.

If $P^{\prime}=\emptyset$, then $p \leq 2\left\lfloor\frac{p}{c+2}+1\right\rfloor \leq \frac{2 p}{c+2}+2$, and hence $\frac{c}{c+2} p \leq 2$. So the length condition on our desired cycle is void, and an arbitrary cycle is provided by Lemma 2.10 .

We may therefore assume that $\left|P^{\prime}\right| \geq 1$; then $S_{1} \cap S_{2}=\emptyset$. If $G$ contains an $S_{1}-S_{2}$ path $Q=s_{1} \ldots s_{2}$ avoiding $P^{\prime}$, then $s_{1} P s_{2} Q s_{1}$ is a cycle of length at least

$$
\left|P^{\prime}\right|+2=p-2\left\lfloor\frac{p}{c+2}+1\right\rfloor+2 \geq p-\frac{2 p}{c+2}=\frac{c}{c+2} p .
$$

If there is no such path $Q$ then $V\left(P^{\prime}\right)$ separates $S_{1}$ from $S_{2}$, so $G$ has a separation $(A, B)$ with $S_{1} \subseteq A \backslash B$ and $S_{2} \subseteq B \backslash A$ and $V\left(P^{\prime}\right)=A \cap B$. But if $|A \backslash B| \leq|B \backslash A|$, say, then the fact that

$$
|A \backslash B| \geq\left|S_{1}\right|>\frac{p}{c+2}
$$

while

$$
|A \cap B|=\left|P^{\prime}\right|=p-2\left\lfloor\frac{p}{c+2}+1\right\rfloor<p-\frac{2 p}{c+2}=c \frac{p}{c+2}
$$

contradicts the $f$-connectedness of $G$ with $f(k) \geq c k$.

Now we are ready to complete the proof of Theorem 2.5.

\section{Proof of Theorem 2.5.}

Our assumptions about $c$ and $n$ imply that $n \geq 3$. By Lemma 2.9, the graph $G$ contains a path of order at least $\frac{c}{c+2} n$. By Lemma 2.11, this implies the existence of a cycle in $G$ of length at least $\left(\frac{c}{c+2}\right)^{2} n$.

\section{$3 \quad$ Forcing factors}

We have shown that graphs satisfying the premise of Conjecture 2.3 contain if not a Hamilton cycle then at least a very long cycle. Another way to relax the statement of Conjecture 2.3 is to ask for a 2-factor instead of a Hamilton cycle (which is just a connected 2-factor).

It turns out that the bound of Conjecture 2.3 does indeed imply the existence of a 2-factor: 
Theorem 3.1 If $f(k) \geq 2 k+1$ for all $k \in \mathbb{N}$, then every $f$-connected graph $G$ of order at least 3 has a 2 -factor.

Slightly smaller $f$-connectedness still forces a 1-factor:

Theorem 3.2 If $f(k) \geq 2 k$ for all $k \in \mathbb{N}$, then every $f$-connected graph $G$ of even order contains a 1-factor.

In contrast to our results for cycles, these two statements are easily seen to be best possible. Indeed, $K_{n, n-1}$ has no 2 -factor but is $f$-connected for $f: k \mapsto 2 k$ when $n$ is odd, while $K_{n, n-2}$ has no 1 -factor but has even order and is $f$-connected for $f: k \mapsto 2 k-1$ when $n$ is odd.

\section{Proof of Theorem 3.2.}

If $G$ has no 1-factor then, by Tutte's 1-factor theorem [12], $G$ has a set $S$ of vertices such that $|S|<k$ for the number $k$ of odd components of $G-S$. Since $G$ has even order, $k$ and $|S|$ have the same parity, so $k \geq|S|+2$.

Let $A$ be the union of the vertex sets of the $\lfloor k / 2\rfloor$ smallest odd components of $G-S$, and let $B$ be the set of all the other vertices of $G-S$. Then $|A| \leq|B|$ but

$$
|S| \leq k-2<2\lfloor k / 2\rfloor \leq 2|A|,
$$

contradicting the $f$-connectedness assumed for $G$.

For 2-factors we use Tutte's f-factor theorem [23, 24] instead of his 1 -factor theorem. (Readable secondary sources for the $f$-factor theorem include [5] and [18].)

\section{Proof of Theorem 3.1.}

By Tutte's $f$-factor theorem, $G=(V, E)$ has a 2-factor if and only if for all disjoint subsets $A, B \subseteq V$

$$
\Theta_{G}(A, B):=2|A|+\sum_{v \in B} d_{G-A}(v)-2|B|-\operatorname{odd}_{G}(A, B) \geq 0 .
$$

Here, $\operatorname{odd}_{G}(A, B)$ denotes the number of odd components with respect to $(A, B)$, which are those components $D$ of $G-A-B$ such that $G$ has an odd number of edges between $B$ and $D$.

Let $G$ be a counterexample to the theorem with as many edges as possible. Since $\Theta_{G}(A, B)$ is clearly always even, there are disjoint $A, B \subseteq V$ such that

$$
\Theta_{G}(A, B) \leq-2
$$


Put $C:=G-A-B$.

If $b \in B$ is adjacent to some other vertex in $B$, then $\Theta_{G}(A, B \backslash\{b\}) \leq$ $\Theta_{G}(A, B)$. Replacing $B$ with a subset if necessary, we may therefore assume that $B$ is an independent set of vertices.

Let us call a component of $C$ even if it is not odd with respect to $(A, B)$. Adding an edge between a vertex in $A$ and any other vertex, or inside a component of $C$, or between two even components, or between an odd and an even component, or between $B$ and an even component will not affect $\Theta(A, B)$. By maximality of $G$ it follows that every vertex $a \in A$ is adjacent to every vertex $v \neq a$ of $G$, that all the components of $C$ are complete, that there is at most one even component, that there is no even component if there is an odd component, and that $B$ is completely joined to every even component.

Let us assume first that there is no odd component. Then two vertices $x, y \in V$ are adjacent if and only if $\{x, y\} \nsubseteq B$, so by (3) we have

$$
2|B| \geq 2|A|+2+p
$$

where $p$ denotes the number of edges between $B$ and $C$. If $|B| \leq 1$ then this gives $|A|=p=0$ and hence $|G| \leq 1$, contrary to our assumption. If $|B| \geq 2$ then $p \geq 2|C|$, and hence

$$
|B| \geq|A|+1+|C|=|V \backslash B|+1 .
$$

Choose a set $X \subseteq B$ of size $\lfloor|B| / 2\rfloor$. Then

$$
|N(X)|=|V \backslash B| \leq|B|-1 \leq 2|X|,
$$

contradicting our assumption that $G$ is $f$-connected with $f(k) \geq 2 k+1$.

We may therefore assume that there is at least one odd component and hence no even component. Let us inductively mark some edges between $B$ and $C$, two at a time, according to the following rules:

(1) As often as possible, mark two edges that are both unmarked and have a common end in $C$. These pairs of edges will be called $\vee$-pairs.

(2) Then, as often as possible, mark any two unmarked edges whose ends in $C$ lie in the same component of $C$.

Then for each component $D$ of $C$ exactly one $B-D$ edge remains unmarked. Thus if $p$ denotes the number of marked pairs, (3) translates to

$$
|B| \geq|A|+1+p .
$$


Write $B$ as a union of two disjoint subsets $B_{1}$ and $B_{2}$, of sizes $\left|B_{1}\right| \leq\left|B_{2}\right|$ as equal as possible. Consider a vertex $x \in C$. Since step (1) of our marking algorithm was performed as often as possible, $x$ has at most one neighbour $y \in B$ such that the edge $x y$ lies in no $\vee$-pair. For every component $D$ of $C$, let $D_{1}$ denote the set of all $x \in D$ for which such a neighbour $y$ exists and lies in $B_{1}$, and define $D_{2}$ correspondingly. Then

$$
D_{1} \cap D_{2}=\emptyset \quad \text { and } \quad\left|D_{1} \cup D_{2}\right|=2 q+1
$$

where $q$ denotes the number of pairs of $B-D$ edges marked in $(2)$. (The +1 comes from the unmarked $B-D$ edge.) Thus, $\left|D_{1}\right| \neq\left|D_{2}\right|$.

Let us define a partial colouring of $V$, as follows. Begin by giving the vertices in $B_{1}$ colour 1 , and those in $B_{2}$ colour 2 . Now consider separately all the components $D$ of $C$.

Suppose first that $\left|D_{1}\right| \geq\left|D_{2}\right|$; then $\left|D_{2}\right| \leq q$. Colour with colour 1 all the vertices in $D_{1}$ that are not adjacent to $B_{2}$. Then any vertex in $D$ that remains uncoloured either lies in $D_{2}$ or is a vertex $x \in D_{1}$ with a neighbour $y \in B_{2}$, in which case $x y$ lies in some $\vee$-pair (because $x \in D_{1}$ ). So the number of vertices in $D$ that remain uncoloured is at most the total number of marked pairs of $B-D$ edges. On the other hand if $\left|D_{1}\right|<\left|D_{2}\right|$, colour with colour 2 all the vertices in $D_{2}$ that are not adjacent to $B_{1}$. As before, the number of vertices in $D$ that remain uncoloured is at most the total number of marked pairs of $B-D$ edges. In total, at most $|A|+p$ vertices in $V$ remain uncoloured, and vertices of distinct colours are nonadjacent.

Let $V_{1}, V_{2} \subseteq V$ be the two colour classes, assume that $\left|V_{1}\right| \leq\left|V_{2}\right|$, and let $U$ be the set of uncoloured vertices. Then $\left|V_{1}\right| \geq\lfloor|B| / 2\rfloor$ (because $V_{1}$ contains either $B_{1}$ or $B_{2}$ ), and $U$ separates $V_{1}$ from $V_{2}$ in $G$. So by $f$ connectedness we have

$$
|A|+p \geq|U| \geq 2\left|V_{1}\right|+1 \geq|B|
$$

contradicting (4).

\section{Other substructures forced by $f$-connectedness}

In this section we list some preliminary observations on what kind of substructures other than long cycles and factors an assumption of $f$-connectedness might force in a graph, together with some open problems that might point towards possible further research. 
Since the idea behind the concept of $f$-connectedness is that it should be a global property, we may ask in particular to what extent it can force substructures that may also occur as global phenomena, such as high chromatic number or large complete minors. As for chromatic number, the only general bound we get follows at once from the fact that $f$-connected graphs for fast growing $f$ cannot have large independent sets of vertices, because dividing such a set into two halves defines a separation. The proof of that bound in fact uses the $f$-connectedness only for one value of $k$ :

Proposition 4.1 Let $t \in \mathbb{N}$, and let $G$ be an $f$-connected graph of order $n$. If $f(k) \geq 2 t(k+1)$ for $k:=\left\lfloor\frac{n}{2(t+1)}\right\rfloor$, then $\chi(G) \geq t+2$.

Proof. Assume that $\chi(G) \leq t+1$. Any $(t+1)$-colouring of $G$ has a colour class $S$ with at least $\frac{n}{t+1} \geq 2 k$ vertices. Choose $S^{\prime} \subseteq S$ with $\left|S^{\prime}\right|=k$. Then $N\left(S^{\prime}\right) \subseteq V(G) \backslash S$, so

$$
\left|N\left(S^{\prime}\right)\right| \leq\left(1-\frac{1}{t+1}\right) n=2 t \frac{n}{2(t+1)}<2 t(k+1) .
$$

This contradicts the $f$-connectedness of $G$, because $S \backslash S^{\prime} \subseteq \overline{S^{\prime}}$ and hence $\left|S^{\prime}\right| \leq\left|S \backslash S^{\prime}\right| \leq\left|\overline{S^{\prime}}\right|$.

In the absence of any restrictions on the graphs considered, Proposition 4.1 cannot be much improved, since the complete $(t+1)$-partite graph with odd partition sets, say of size $2 s+1$, is $f$-connected with $f(k)=$ $t(2 k+1)$. An assumption of large girth may help to force the chromatic number up, but since there are bipartite expanders of order $n$ and girth $\Theta(\log n)[19]$, this will still require that $f$ grows linearly.

Friedman and Pippenger [16] proved (a result implying) that, given $d, t \in$ $\mathbb{N}$, every graph satisfying $|N(S)| \geq(d+1)|S|$ for all sets $S$ of at most $2 t-2$ vertices contains every tree of maximum degree at most $d$ and order at most $t$ as a subgraph. In terms of $f$-connectedness, this implies the following:

Proposition 4.2 Let $d \in \mathbb{N}$, and let $G$ be an $f$-connected graph or order $n$ with $f(k) \geq(d+1) k$ for all $k \in \mathbb{N}$. Then $G$ contains every tree of maximum degree at most $d$ and order $t \leq \frac{n}{2 d+6}+1$.

Proof. By Friedman and Pippenger's theorem, it suffices to show that $|N(S)| \geq(d+1)|S|$ for all $S \subseteq V(G)$ with

$$
|S| \leq 2 t-2 \leq \frac{n}{d+3} .
$$


By (1), the inequality $|N(S)| \geq(d+1)|S|$ can fail only if

$$
|N(S)| \geq n-2|S|+1 \underset{(5)}{\geq}(d+1)|S|+1,
$$

so it holds for every $S$ as in (5).

The occurrence of a given subgraph will hardly count as a global phenomenon. But for the record let us estimate the growth rate of $f$ needed to force a $K_{r}$ subgraph by $f$-connectedness. Splitting a largest independent set in half as in Proposition 4.1, we can apply Ramsey's theorem to obtain a trivial upper bound in terms of $R(r, s)$, the smallest integer $n$ such that every graph of order $n$ contains either a $K_{r}$ or $s$ independent vertices:

Lemma 4.3 If $f$ satisfies $f(k) \geq R(r, 2 k+2)-2 k$ for all $k \in \mathbb{N}$, then every $f$-connected graph has a $K_{r}$ subgraph.

Proof. Let $G$ be an $f$-connected $K_{r}$-free graph. Let $S$ be a largest independent set of vertices and put $|S|=: \alpha$. Pick $S^{\prime} \subseteq S$ with $\left|S^{\prime}\right|=\left\lfloor\frac{\alpha}{2}\right\rfloor=: k$. Then

$$
f(k) \leq\left|N\left(S^{\prime}\right)\right| \leq n-2 k<R(r, 2 k+2)-2 k,
$$

since $G$ is $K_{r}$-free with independence number $\alpha \leq 2 k+1$.

With the Erdös-Szekeres bound [14] of

$$
R(r, s)=R(s, r) \leq\left(\begin{array}{c}
s+r-2 \\
s-1
\end{array}\right)
$$

for $R(r, s)$, Lemma 4.3 for $s=2 k+2$ implies that polynomial growth of $f$ suffices to force a $K_{r}$ subgraph for fixed $r$ :

Proposition 4.3 For every $r \in \mathbb{N}$ there is a polynomial $p_{r}(k)=O\left(k^{r-1}\right)$ such that every $p_{r}$-connected graph has a $K_{r}$ subgraph.

For $r=3$ we can do a little better by Kim's theorem [17], which implies that $p_{3}(k)=O\left(k^{2} / \log k\right)$. But note that even this bound for $p_{3}$ is hardly smaller than $2(k+1)^{2}$, which by Theorem 2.1 already forces a Hamilton cycle.

In contrast to complete subgraphs, dense minors can occur in sparse graphs as a global phenomenon. It would thus seem that forcing large complete minors (say) might be a likely feat of $f$-connectedness which other 
invariants cannot as easily achieve. However, this is not the case. As grids are about $\sqrt{ }$-connected but have no $K_{5}$ minor, no function

$$
f(k)=o\left(k^{1 / 2}\right)
$$

could achieve this. On the other hand, Alon, Seymour and Thomas [2] proved that every $n$-vertex graph $G$ without a $K_{r}$ minor has a separation $(A, B)$ with $|A \cap B| \leq r^{3 / 2} n^{1 / 2}$ and $|A \backslash B| \leq|B \backslash A| \leq 2 n / 3$. Then $|A \backslash B| \geq n-r^{3 / 2} n^{1 / 2}-2 n / 3 \geq n / 4$ if $n$ is large enough, in which case $G$ will not be $f$-connected for any $f$ with $f(\lceil n / 4\rceil)>r^{3 / 2} n^{1 / 2}$.

Thus if $H$ is any fixed graph and

$$
f(k)=\omega\left(k^{1 / 2}\right),
$$

then all large enough $f$-connected graphs have an $H$ minor. The problem that remains is the following:

Problem 4.4 Is there a function $f(k)=O\left(k^{1 / 2}\right)$ such that, given any graph $H$, every large enough $f$-connected graph has an $H$ minor?

We expect the answer to be negative, ie. that for every $c \in \mathbb{R}$ there exist an $r \in \mathbb{N}$ and arbitrarily large $c \sqrt{ }$-connected graphs that have no $K_{r}$ minor.

More positively, one might speculate whether all graphs that are about $\sqrt{ }$-connected look like grids:

Problem 4.5 Given constants $0<c<d$ and $s \in \mathbb{N}$, is every $c \sqrt{ }$-connected graph that has no $d \sqrt{ }{ }^{-}$-connected subgraph of order at least s grid-like in some well-specified sense (depending on $c$, $d$ and $s$ )?

See [13] for some ideas on how grid-likeness might be specified.

A graph that is highly connected in some global sense ought to have large tree-width. Our next observation confirms this for $f$-connectedness: any reasonable bound $g(n)$ on the tree-width of an $n$-vertex graph is exceeded by the tree-width of every large enough $f$-connected graph, as soon as $f=\omega(g)$.

We begin with a technical lemma that uses a standard technique to obtain a lower tree-width bound:

Lemma 4.6 If $f, g$ are non-decreasing $\mathbb{R}^{+} \rightarrow \mathbb{R}$ functions and $n \in \mathbb{N}$ is such that $f\left(\frac{1}{3}(n-g(n))\right)>g(n)$, then every $f$-connected graph of order $n$ has tree-width at least $g(n)$. 
Proof. Let $G$ be a graph of order $n$ with a tree-decomposition $\mathcal{D}$ of width less than $g(n)$, ie. into parts of size at most $g(n)$. Assume without loss of generality that no part of $\mathcal{D}$ contains any other part. We show that $G$ cannot be $f$-connected.

Every edge $e$ of the decomposition tree $T$ of $\mathcal{D}$ induces a separation of $G$. We orient $e$ towards the larger side of that separation, breaking ties arbitrarily. Then a sink node $t$ of $T$ corresponds to a part $V_{t}$ of $\mathcal{D}$ such that either $G-V_{t}$ has a component $C$ of order at least $\frac{1}{3}\left|G-V_{t}\right|$ (but no bigger than $|G-C-N(C)|$ ), or else there is a suitable union $C$ of components of $G-V_{t}$ such that

$$
\frac{1}{3}\left|G-V_{t}\right| \leq|C| \leq \frac{1}{2}\left|G-V_{t}\right| .
$$

As $\left|V_{t}\right| \leq g(n)$, we thus have $\frac{1}{3}(n-g(n)) \leq|C|$. As $N(C) \subseteq V_{t}$, this yields

$$
|N(C)| \leq g(n)<f\left(\frac{1}{3}(n-g(n))\right) \leq f(|C|) .
$$

Therefore $G$ is not $f$-connected.

Lemma 4.6 can be applied as follows. Consider any 'reasonable' bound $g(n)$ on the tree-width of an $n$-vertex graph $G$. Normally, we will have $g(n)=o(n)$, and may therefore expect $g$ to be concave, ie. to satisfy

$$
g(x+\lambda(y-x)) \geq g(x)+\lambda(g(y)-g(x))
$$

whenever $x<y$ and $\lambda \in(0,1)$.

Proposition 4.6 Let $f, g$ be two non-decreasing $\mathbb{R}^{+} \rightarrow \mathbb{R}$ functions such that $f=\omega(g)$ and $g(n)=o(n)$. If $g$ is concave, then every $f$-connected graph of large enough order $n$ has tree-width at least $g(n)$.

Proof. Since $g(n)=o(n)$, we have $\frac{1}{3}(n-g(n))>n / 4$ for $n$ large enough. In order to deduce the assertion from Lemma 4.6, it thus suffices to show that $f(n / 4)>g(n)$ for $n$ large.

It is not difficult to show that, since $g$ is concave, $g(n)=o(n)$ implies that $g(n)<4 g(n / 4)$ for all sufficienly large $n$. Hence for $n$ large,

$$
\frac{g(n) / 4}{f(n / 4)}<\frac{g(n / 4)}{f(n / 4)}<\frac{1}{4}
$$

because $f=\omega(g)$, and hence $g(n)<f(n / 4)$ as desired. 
A graph $G$ is called $t$-tough if for every separating set $S \subseteq V(G)$ the graph $G-S$ has at most $|S| / t$ components. While it is easy to see that a graph with a Hamilton cycle is 1-tough, Chvátal [10] conjectured that there is a constant $t$ such that every $t$-tough graph is hamiltonian. It is known [4] that $t$ cannot be less than $9 / 4$.

Our next result implies that if Chvátal's conjecture is true, with $t=t_{0}$ say, then every $f$-connected graph with $f(k) \geq 2 t_{0}(k+1)$ is hamiltonian. (Our Conjecture 2.2 can thus be viewed as a relaxation of Chvátal's conjecture.)

Proposition 4.7 Let $t \in \mathbb{N}$. If $f(k) \geq 2 t(k+1)$ for all $k \in \mathbb{N}$, then every f-connected graph is t-tough.

Proof. Suppose $G$ is not $t$-tough, and let $S$ be a separating set of $k$ vertices whose deletion results in more than $k / t$ components. Let $A$ be the union of $S$ and the smallest $\lfloor k / 2 t\rfloor$ of those components. Then taking $B$ to be the union of $S$ and the other components makes $(A, B)$ into a separation in which $A$ is the smaller side and $|A \backslash B| \geq\lfloor k / 2 t\rfloor$. Since $f(\lfloor k / 2 t\rfloor)>k$, the fact that $|A \cap B|=|S|=k$ means that $G$ is not $f$-connected.

Thus, suitable linear $f$-connectedness implies any constant toughness. The converse, of course, is far from true: identifying two large complete graphs $K_{r}$ in $2 t$ vertices yields a $t$-tough graph that will not be $f$-connected for any given $f \rightarrow \infty$ if $r$ is large enough.

\section{$5 \quad$ Expanding graphs and $f$-connectedness}

The expansion of a graph can be estimated in terms of the eigenvalues of certain matrices associated with this graph. In this section we relate $f$ connectedness for a linear function $f$ to expansion and we determine a linear lower bound for the function $f$ for a regular graph in terms of the eigenvalues of its adjacency matrix. Combined with our results from Sections 2 and 3 this will be applied to show the existence of cycles and factors in so-called Ramanujan graphs.

There are several concepts measuring the expansion of a graph. One of the earliest of them is that of an $(n, d, c)$-expander. Let $G=(V, E)$ be a $d$ regular graph of order $n$, and let $c \in(0,1] . G$ is called an $(n, d, c)$-expander [3] if for every set $X \subseteq V(G)$ with $|X| \leq \frac{n}{2}$ we have $|N(X)| \geq c|X|$. Since $|A| \leq \frac{n}{2}$ if $|A| \leq|\bar{A}|$, every $(n, d, c)$-expander is $f$-connected for $f(k)=c k$. Conversely, we have the following: 
Proposition 5.1 If $G$ is $f$-connected with $f(k) \geq c k$, then $G$ is an $\left(n, d, c^{\prime}\right)$ expander with $c^{\prime}=\frac{c}{c+1}$.

Proof. Let $A \subseteq V(G)$ with $|A| \leq \frac{n}{2}$ be given. If $|A| \leq|\bar{A}|$, then $|N(A)| \geq$ $c|A| \geq c^{\prime}|A|$ as required. If not, then $|\bar{A}|<|A|$ and hence $|N(\bar{A})| \geq c|\bar{A}|$. Since $N(\bar{A}) \subseteq N(A)$ and $n-|A| \geq|A|$, we obtain

$$
|N(A)| \geq|N(\bar{A})| \geq c|\bar{A}|=c(n-|A|-|N(A)|) \geq c(|A|-|N(A)|)
$$

and hence $|N(A)| \geq \frac{c}{c+1}|A|=c^{\prime}|A|$.

Let $d=\lambda_{1} \geq \lambda_{2} \geq \ldots \geq \lambda_{n}$ be the spectrum of the adjacency matrix of $G$, and let

$$
\lambda:=\max \left\{\left|\lambda_{2}\right|,\left|\lambda_{n}\right|\right\}
$$

be the largest absolute value of an eigenvalue other than $\lambda_{1}$.

Lemma 5.2 Every d-regular graph $G$ is $f$-connected with $f(k) \geq\left(\frac{d}{\lambda}-1\right) k$.

In our proof of Lemma 5.2 we will use the following inequality for $d$ regular graphs [3, Theorem 9.2.4]: for all $S \subseteq V$ and $s:=|S|$,

$$
\sum_{v \in V}\left(|N(v) \cap S|-\frac{d s}{n}\right)^{2} \leq \lambda^{2} \frac{s(n-s)}{n} .
$$

\section{Proof of Lemma 5.2.}

Choose a set $S \subseteq V$ with $s:=|S| \leq|\bar{S}|=: \bar{s}$ such that

$$
c:=\frac{|N(S)|}{|S|}
$$

is minimum. Then $G$ is $f$-connected with $f(k)=c k$, so all we have to show is that $c \geq \frac{d}{\lambda}-1$. Note that

$$
\frac{n-s}{\bar{s}}=\frac{|N(S)|}{|\bar{S}|}+1 \leq \frac{|N(S)|}{|S|}+1=c+1=\frac{|S|+|N(S)|}{|S|}=\frac{n-\bar{s}}{s} .
$$

By definition of $\bar{S}$ we have $N(v) \cap S=\emptyset$ for every $v \in \bar{S}$. Double counting gives

$$
\sum_{v \in V \backslash \bar{S}}|N(v) \cap S|=2|E(G[S])|+|E(S, N(S))|=\sum_{v \in S} d(v)=d s .
$$


Thus,

$$
\begin{aligned}
\sum_{v \in V}\left(|N(v) \cap S|-\frac{d s}{n}\right)^{2} & =\sum_{v \in \bar{S}}\left(|N(v) \cap S|-\frac{d s}{n}\right)^{2}+\sum_{v \in V \backslash \bar{S}}\left(|N(v) \cap S|-\frac{d s}{n}\right)^{2} \\
& \geq \bar{s}\left(\frac{d s}{n}\right)^{2}+(n-\bar{s})\left(\frac{d s}{n-\bar{s}}-\frac{d s}{n}\right)^{2} \\
& =\left(\frac{d s}{n}\right)^{2} \frac{n \bar{s}}{n-\bar{s}},
\end{aligned}
$$

where the inequality follows from the fact that a sum of squares of terms with constant sum is minimized if all summands are equal. Combining this with (6) and using (7) we obtain

$$
\left(\frac{d}{\lambda}\right)^{2} \leq \frac{n-\bar{s}}{s} \cdot \frac{n-s}{\bar{s}} \leq(c+1)^{2}
$$

and hence $c \geq \frac{d}{\lambda}-1$, as desired.

Recall that the eigenvalues $\lambda_{i}$ of a $d$-regular graph satisfy

$$
d=\lambda_{1} \geq \ldots \geq \lambda_{n} \geq-d \text {. }
$$

The graph is bipartite if and only if $\lambda_{n}=-d$; it is connected if and only if $d=\lambda_{1}>\lambda_{2}$ (see eg. [11]). The expansion of a graph is frequently estimated in terms of $\lambda_{2}$ or the value $\lambda=\max \left\{\left|\lambda_{2}\right|,\left|\lambda_{n}\right|\right\}$ considered above. Alon and Boppana (see Alon [1]) observed that for every infinite family $G_{1}, G_{2}, \ldots$ of $d$-regular graphs the second-largest eigenvalues satisfy

$$
\liminf \lambda_{2}\left(G_{i}\right) \geq 2 \sqrt{d-1} .
$$

Although relatively sparse random graphs are well-expanding, the explicit construction of infinite sequences of sparse expanding graphs turned out to be difficult. Lubotzky, Phillips and Sarnak [19] achieved a breakthrough by constructing, for infinitely many values of $d$, infinite sequences of connected $d$-regular graphs that satisfy $\left|\lambda_{i}\right| \leq 2 \sqrt{d-1}$ for all $i$ with $\lambda_{i} \neq \pm d$, which is best possible by (8). They called graphs with this property Ramanujan graphs, because the algebraic construction of these graphs is related to a Ramanujan conjecture. Some of the sequences of Ramanujan graphs that they constructed are bipartite (thus $\lambda_{n}=-d$ ) and some are non-bipartite. The latter graphs satisfy $\lambda \leq 2 \sqrt{d-1}$ and hence they are $f$-connected for a linear function $f$ by Lemma 5.2 . 
Lubotzky, Phillips and Sarnak [19] observed that the graphs that they constructed have large girth and small independence number compared with the order of the graph. Combining Lemma 5.2 with Theorems 2.4 and 2.5 we get that non-bipartite Ramanujan graphs with $d \geq 3$ have a cycle of length linear in $n$. For larger $d$ they even have factors and cycles of length close to $n$.

Corollary 5.3 Let $G$ be a non-bipartite d-regular Ramanujan graph of order $n$. If $d \geq 35$, then $G$ contains a 1-factor (if $n$ is even), a 2-factor, and a cycle of length at least $\frac{d}{d+2 \sqrt{d-1}} n>\frac{3}{4} n$. If Conjecture 2.3 is true, then $G$ is hamiltonian.

Proof. Since $G$ is connected and non-bipartite we have $\lambda<d$, and hence $\lambda \leq 2 \sqrt{d-1}$ as $G$ is a Ramanujan graph. For $d \geq 35$ this implies $d / \lambda \geq$ $d /(2 \sqrt{d-1})>3$. By Lemma 5.2, therefore, $G$ is $f$-connected with $f(k) \geq$ $\left\lceil\left(\frac{d}{\lambda}-1\right) k\right\rceil \geq 2 k+1$. The assertions now follow from our Theorems 3.2, 3.1 and 2.4 .

\section{References}

[1] N. Alon, Eigenvalues and expanders, Combinatorica 6 (1986), 83-96.

[2] N. Alon, P. Seymour, and R. Thomas, A separator theorem for non-planar graphs, J. Amer. Math. Soc. 3 (1990), 801-808.

[3] N. Alon and J. Spencer, The Probabilistic Method, Wiley 1992.

[4] D. Bauer, H. J. Broersma and H. J. Veldman, Not every 2-tough graph is hamiltonian, Discrete Appl. Math. 99 (2000), 317-321.

[5] B. Bollobás, Extremal Graph Theory, Academic Press 1978.

[6] B. Bollobás and I.B. Leader, Compressions and isoperimetric inequalities J. Combin. Theory A 56 (1991), 47-62.

[7] J.A. Bondy, Basic Graph Theory: Paths and Circuits, in: Handbook of Combinatorics, Vol. I, Elsevier, Amsterdam, 1995, 3-110.

[8] A. Brouwer, Toughness and spectrum of a graph, Linear Algebra Appl. 226-228 (1995), 267-271. 
[9] F.R.K. Chung, Spectral Graph Theory, American Mathematical Society 1997.

[10] V. Chvátal, Tough graphs and hamiltonian cycles, Discrete Math. 5 (1973), 215-228.

[11] D.M. Cvetkovic, M. Doob, and H. Sachs, Spectra of Graphs (3rd edition), Johann Ambrosius Barth Verlag, Heidelberg, Leipzig, 1995.

[12] R. Diestel, Graph Theory (2nd edition), Springer-Verlag 2000. http://www.math. uni-hamburg.de/home/diestel/books/graph.theory/download.html

[13] R. Diestel and D. KüHn, Graph minor hierarchies, preprint 2001.

[14] P. Erdős and G. Szekeres, A combinatorial problem in geometry, Compos. Math. 2 (1935), 463-470.

[15] J. Friedman, ed., Expanding Graphs, American Mathematical Society 1993.

[16] J. Friedman and N. Pippenger, Expanding graphs contain all small trees, Combinatorica 7 (1987), 71-76.

[17] J. H. KIm, The Ramsey number $R(3, t)$ has order of magnitude $t^{2} / \log t$, Random Structures \& Algorithms 7 (1995), 173-207.

[18] L. Lovász and M.D. Plummer, Matching Theory, Annals of Discrete Math. 29, North Holland 1986.

[19] A. Lubotzky, R. Phillips And P. Sarnak, Ramanujan graphs, Combinatorica 8 (1988), 261-277.

[20] L. PósA, Hamiltonian circuits in random graphs, Discrete Math. 14 (1976), 359-364.

[21] B.A. REED, Tree width and tangles: a new connectivity measure and some applications, in (R.A. BAILEy, ed.): Surveys in Combinatorics 1997, Cambridge University Press 1997, 87-162.

[22] C. Rempel, Erzwingung von Teilstrukturen in Graphen durch globale Parameter, Diss. Univ. Hamburg 2001.

[23] W.T. Tutte, The factors of a graph, Canadian J. Math. 4 (1952), $314-328$. 
[24] W.T. TutTe, A short proof of the factor theorem for finite graphs, Canadian J. Math. 6 (1954), 347-352. 